\title{
Correction to: Multiplayer Pursuit-Evasion Games in Three-Dimensional Flow Fields
}

\author{
Wei Sun ${ }^{1}$ (D) Panagiotis Tsiotras ${ }^{2} \cdot$ Anthony J. Yezzi ${ }^{3}$ \\ Published online: 22 March 2019 \\ ○) Springer Science+Business Media, LLC, part of Springer Nature 2019
}

\section{Correction to: Dynamic Games and Applications https://doi.org/10.1007/s13235-019-00304-4}

Unfortunately, the acknowledgement text was not submitted during the acceptance of the manuscript. Hence, it was not included in the original publication of the article. This has been corrected with this erratum.

Acknowledgements This work has been supported by NSF award CMMI-1662542, NSF award CCF-1526848 and ARO award W911NF-18-1-0281.

Publisher's Note Springer Nature remains neutral with regard to jurisdictional claims in published maps and institutional affiliations.

The original article can be found online at https://doi.org/10.1007/s13235-019-00304-4.

$\triangle$ Wei Sun

wsun@ou.edu

Panagiotis Tsiotras

tsiotras@gatech.edu

Anthony J. Yezzi

ayezzi@ece.gatech.edu

1 School of Aerospace and Mechanical Engineering, University of Oklahoma, Norman, OK 73019, USA

2 School of Aerospace Engineering and Institute for Robotics and Intelligent Machines, Georgia Institute of Technology, Atlanta, GA 30332, USA

3 School of Electrical and Computer Engineering, Georgia Institute of Technology, Atlanta, GA 30332, USA 